\title{
A Molecular Aspect in the Regulation of Drug Metabolism: Does PXR-Induced Enzyme Expression Always Lead to Functional Changes in Drug Metabolism?
}

\author{
Yuan Wei ${ }^{1} \cdot$ Chenxiao Tang ${ }^{1} \cdot$ Vinayak Sant $^{1} \cdot \operatorname{Song} \mathrm{Li}^{1}$. \\ Samuel M. Poloyac ${ }^{1} \cdot$ Wen Xie $^{1}$
}

Published online: 4 May 2016

(C) Springer International Publishing AG 2016

\begin{abstract}
Pregnane X receptor (PXR, NR112) is a xenobiotic receptor whose primary function is to regulate the expression of drug-metabolizing enzymes (DMEs) and drug transporters. Drug-induced PXR activation and subsequent enzyme and transporter induction has been proposed to be an important mechanism for the drug-drug interactions. In addition to activating PXR, many pharmaceutical chemicals can also function as reversible or irreversible inhibitors of DMEs, which may also impact the pharmacokinetics and pharmacodynamics (PK/PD) of drugs. Therefore, we cannot simply conclude that the PXR-induced alteration in enzyme expression always reflects functional changes. We should consider both PXR activation and DMEs inhibition to improve drug safety in the clinic.
\end{abstract}

Keywords Drug metabolism $\cdot$ Gene regulation $\cdot$ Nuclear receptor $\cdot$ Enzyme inhibition

\section{PXR-Mediated Transcriptional Regulation of Drug-Metabolizing Enzymes (DMEs)}

\section{An Overview of DMEs}

The biotransformation (metabolism) of xenobiotics including drugs is widely accepted to be divided into phase I and phase

This article is part of the Topical Collection on Molecular Drug Disposition

Wen Xie

wex6@pitt.edu

1 Department of Pharmaceutical Sciences, School of Pharmacy, University of Pittsburgh, 306 Salk Pavilion, 335 Sutherland Drive, Pittsburgh, PA 15261, USA
II metabolism. Phase I metabolism includes oxidation, reduction, hydrolysis, and hydration reactions which can convert parent drugs into more polar and water soluble metabolites [1]. Cytochrome P450 enzymes (CYPs) are the primary enzymes involved in the phase I metabolism. The P450 enzymes belong to a superfamily of heme-thiolate proteins that are responsible for catalyzing monooxygenase reactions of lipophilic compounds, which is facilitated by the reducing power of the NADPH P450 oxidoreductase [2]. CYPs add hydroxyl groups onto the parent drug to biotransform the hydroxyl groups and biotransform xenobiotics into more polar and more readily excreted metabolites, which may or may not be utilized by the phase II enzymes for further conjugation and detoxification. Phase II metabolism, also called conjugation reactions includes glucuronidation, sulfation, methylation, acetylation, glutathione, and amino acid conjugation [3]. These phase II reactions are mainly catalyzed by transferases, such as the UDP-glucuronosyltransferases (UGTs), sulfotransferases (SULTs), methyltransferase, $N$-acetyltransferases, and glutathione $S$-transferases (GSTs). Although DMEs are primarily responsible for drug metabolism and disposition, they can also be involved in drug-drug interactions and may cause toxic side effects of drugs. In a typical drugdrug interaction, the induction of DMEs by a drug can influence the metabolism of other drugs taken by the same patient if the other drugs happen to be the substrates of the same enzyme(s) that have been induced.

\section{PXR as a Xenobiotic Receptor that Regulates DMEs}

Pregnane X receptor (PXR, NR112) was independently cloned in the laboratories of Steve Kliewer, then at Glaxo Wellcome [4] and Ron Evans at the Salk Institute [5]. The Kliewer lab found the mouse PXR from a gene fragment in the Washington University Mouse Expressed-Sequence Tag 
(EST) Database [4], whereas the Evans lab cloned the human PXR, also termed SXR or steroid and xenobiotic receptor, as a homolog of the Xenopus benzoate X receptors (BXR) [6]. The discovery of PXR benefited from earlier work published by Phil Guzelian's laboratory at the University of Colorado, who suggested that there are cellular factor and a defined DNA element responsible for drug responsive regulation of the human and rodent CYP3A genes in hepatocytes [7]. The cellular factor turned out to be PXR and the DNA element turned out to be the PXR response element in the CYP3A gene promoter. CYP3A is a prototypical target gene of PXR. PXR was named based on its activation by the pregnanes 21 -carbon steroids [8]. Since the initial cloning of the mouse and human PXR, the cloning of PXR in different species including mammals, birds, and fish have been reported by various groups [9]. Similar to many other typical nuclear receptors, PXR contains a double zinc-finger DNA-binding domain (DBD) and a carboxyterminal ligand-binding domain (LBD). It has a relatively short hinge region (amino acids 107-141) that separates the DBD and LBD [10]. The PXR structure also contains some major differences compared with other nuclear receptors. The spherical ligand-binding pocket of PXR is at least twice as large as other steroid hormone- or retinoid receptor ligandbinding pockets and also because of its extremely hydrophobic and flexible properties, PXR allows the recognition of a wide range of xenobiotics $[11,12]$.

Early functional characterization revealed that one of the primary PXR functions is the transcriptional regulation of DMEs. Binding of PXR to the PXR-responsive elements (PXREs) in the target gene promoters usually leads to the induction of DMEs, notably the CYP3A and CYP2B subfamilies. In addition, UGTs, SULTs, and drug transporters such as the organic-anion transporter 2 (OATP2), multidrug resistance 1 (MDR1), and multidrug resistance-associated protein 2 (MRP2) were also shown to be transcriptionally regulated by PXR $[8,13]$. Examples of the PXREs include DR-3, DR4 , and IR-6 identified in the promoter regions of CYP3A, CYP2B, and CYP2C genes [1]. Among the PXR target genes, CYP3A and CYP2B subfamilies are the most functionally relevant in drug/xenobiotic metabolism, because they are involved in the elimination of xenobiotics and endogenous compounds and, they are often involved in the clinically significant drug-drug interactions [14]. The expression of CYP enzymes and transporters can be induced by xenobiotic drugs, such as rifampicin, phenobarbital, dexamethasone, and the herbal medicine St John's wort. PXR is also involved in and has a complex effect on cholesterol hemostasis and bile acid metabolism [15]. Besides its expression in normal tissues, PXR also has a role in cancer tissues, such as breast cancer, ovarian cancer, and prostate cancer [16]. Studies over the past 17 years have clearly established PXR as a xenosensor and master regulator of xenobiotic responses [2]. More recently, it has become clear that the nuclear receptor-mediated regulation of enzymes and transporters can not only impact drug metabolism, but also influence many physiological and disease pathways by affecting the homeostasis of endogenous chemicals, such as the bile acids, bilirubin, steroid hormones, glucose, and lipids.

\section{Inhibition of DME}

Hepatic cytochrome P450 enzymes are major drugmetabolizing enzymes and represent a major pathway of drug metabolism. The superfamily of CYP450 isoforms has a broad spectrum of substrates. CYP inhibitions are clinically relevant to drug metabolism and drug-drug interactions, because they can potentially alter the PK/PD of drugs, especially in the practice of polypharmacy where patients take multiple medications. Among examples, the calcium-channel blocker Mibefradil was withdrawn from the market in 1998 due to mechanism-based inhibition of CYP3A4 through the destruction of heme moiety, which causes a significant increase in the plasma concentration of co-administered drugs [18, 19].

The CYP inhibition can be generally divided into three categories: reversible inhibition, irreversible inhibition, and quasi-irreversible inhibition $[17,20]$. The two types of irreversible inhibitions are sometimes also referred to as mechanism-based inhibition [21-24]. The reversible inhibition is the most common reason for documented drug-drug interactions $[20,25]$. Reversible inhibition occurs between the competition for CYP binding site between substrate drugs and inhibitors. Reversible inhibition can be further divided into competitive, non-competitive, uncompetitive, and mixed-type inhibition $[21,23,25]$. Competitive inhibition happens between mutually exclusive substrates and inhibitors. A non-competitive inhibitor often binds to another enzyme active site or enzyme-substrate complex [23]. Mixed inhibition is a combination of competitive inhibition and non-competitive inhibition $[20,23]$. In contrast to the non-competitive inhibitors, uncompetitive inhibitors only bind to an enzyme-substrate complex but not another enzyme active site. The four types of enzyme inhibitions can be differentiated by monitoring the $K_{\mathrm{m}}$ and $V_{\max }$ values of the substrates, as summarized in Table 1. Many reversible inhibitors contain nitrogen, including imidazoles, pyridines, quinolines, and triazoles [20, 26]. Examples of the reversible inhibitor drugs include azole antifungals, cimetidine, metyrapone, HIV protease inhibitor, ellipticine, quinidine, and antimalarial agents [24]. In some cases, an inhibitor can exhibit multiple modes of inhibition toward different CYP isoforms. Dihydrotanshinone, found in the Chinese herb Danshen, is a competitive inhibitor of human CYP1A2, CYP2C9, a non-competitive inhibitor of CYP3A4, and an uncompetitive inhibitor of CYP2E1 [27].

Compared with the reversible inhibition, irreversible inhibitions are more likely to result in unfavorable inhibition [28]. 
Table 1 Kinetics of reversible inhibition

\begin{tabular}{lllll}
\hline & $\begin{array}{l}\text { Competitive } \\
\text { inhibitor }\end{array}$ & $\begin{array}{l}\text { Non-competitive } \\
\text { inhibitor }\end{array}$ & $\begin{array}{l}\text { Uncompetitive } \\
\text { inhibitor }\end{array}$ & Mixed-type inhibitor \\
\hline$K_{\mathrm{m}}$ & Increased & Unchanged & Decreased & Increased or decreased \\
$V_{\max }$ & Unchanged & Decreased & Decreased & Decreased \\
\hline
\end{tabular}

For irreversible inhibition, compounds are metabolized by $\mathrm{P} 450$ s to their intermediates, which cause the irreversibility of CYP inhibition until the new CYP enzymes are synthesized. Drugs that can cause the irreversibility of CYP enzymes are called mechanism-based drug inactivators (MBI) [17]. Inhibitors that covalently bind with amino acid at active site or heme nitrogen of CYP enzyme are considered true irreversible inhibitors. Quasi-irreversible inhibitors can coordinate with heme iron to form metabolic intermediates, which occupy the active site of CYP enzyme, leading to the inhibitory effect. Quasi-irreversible inhibition could be reversed by three methods in vitro: incubation with highly lipophilic compounds; irradiation at 400-500 nm; or addition of potassium ferricyanide [20, 29, 30]. In vivo, the binding of metabolite intermediates with CYP enzyme is very stable, which cannot be reversed [20]. In order to regain the capability of CYP enzymes, new enzymes need to be synthesized.

There are several mechanisms for the irreversible inhibition. The first mechanism is that the reactive intermediates alkylate heme, resulting in the heme destruction [20, 29, 31, 32]. For example, secobarbital, gestodene, spiro- and dispiro1,2,4-trioxolane, and Gemfibrozil all can cause mechanismbased inhibition through heme alkylation [33-36]. In the second mechanism, the reactive intermediates covalently bind to amino acid residues within the apoprotein [29], in which drugs containing sulfur groups or nitrogen groups can be oxidized by CYP enzyme and subsequently modify the CYP proteins $[20,29,30]$. Among examples, diethyldithocarbamate, a metabolite of disulfiram, exhibits MBI through covalent binding to the CYP2E1 apoprotein [37]. Also, the anti-progestin Mifepristone inactivates CYP3A4 by irreversible modification of apoprotein at the enzyme active site [38]. In addition, some compounds are capable of changing heme and apoprotein simultaneously. The covalent binding of the modified heme to the apoprotein can cause MBI [39]. For example, $17 \alpha$-ethynylestradiol, a common constituent of oral contraceptives, can inactivate CYP3A4 through covalent attachment of the modified heme to the apoprotein [39].

Mechanism-based inhibitions have received increasing attention in the clinic, because they might cause prolonged clearance of the inactivator drugs and other drugs that are metabolized by the same CYP enzymes, which cause drugdrug interactions, and this effect is long lasting compared with the reversible inhibition. There are several therapeutic drugs that are known as mechanism-based inhibitors. Among examples, Yamaori and colleagues found that cannabinoid (CBD) is the mechanism-based inactivator of CYP1A1. The inactivation might be the result of the formation of an electrophilic quinone from the methylresorcinol moiety of CBD by the human CYP1A1 [40]. Lu and colleagues reported that zileuton is a mechanism-based inhibitor of CYP1A2 [41]. Imperatorin and isoimperatorin showed irreversible inhibition of CYP2B6 by forming the $\gamma$-ketoenal intermediate [42, 43]. It has also been reported that the antibacterials isoniazid and clarithromycin, anticancer agents lapatinib and tamoxifen, antihypertensives diltiazem, nicardipine and verapamil, antiHIV agents ritonavir and delavirdine, and carbamazepine can act as inactivators for CYP3A4 [44, 45]. In addition to drugs, some food or food components can also act as the mechanismbased inactivators. Sunaga and colleagues reported that tomato juice extract can cause mechanism-based inhibition of the hepatic and intestinal CYP3A4 [46]. Greenblatt and colleagues reported that grapefruit juice exhibited mechanismbased inhibition of CYP3A4 [47].

\section{Does PXR Activation Always Cause Increased Drug Metabolism?}

PXR is widely accepted as the principal transcriptional regulator of CYP3A induction by xenobiotics [48, 49]. PXR can be activated by a variety of natural and synthetic compounds. PXR induces CYP gene expression by binding as heterodimers with the 9-cis retinoic acid receptor $\alpha(\mathrm{RXR} \alpha)$ to DR3, DR4, and ER6 motif in the proximal promoter region of CYP3A4 gene [9]. Due to its abundance in the liver and small intestine, CYP3A4 is responsible for metabolizing more than $50 \%$ of the clinical drugs. CYP3A4 is highly inducible, so its activation usually accelerates the metabolism of other drugs and thus causes drug-drug interactions [50]. Sinz and colleagues investigated 170 xenobiotics to study the relationship between human PXR and CYP3A4. They compared the compounds with Rifampicin (RIF), an effective inducer of human CYP3A4 and a typical PXR activator [9] and concluded that a compound, which transactivates human PXR more than $40 \%$ of the RIF response, has a high potential for inducing CYP3A4. Studies about RIF and its activation of PXR continued to grow in recent years. RIF and isoniazid (INH) are the major drugs used to treat tuberculosis. However, a combination of these two drugs frequently causes liver injury [51]. It has been reported that the PXR-humanized mice showed 
higher amounts of serum alanine aminotransferase and alkaline phosphatase activity after co-treatment with RIF and INH.

Although it is generally believed that activation of PXR will increase drug metabolism, this is not always the case. Wu Wei Zi (WWZ, or Schisandra sphenanthera), a traditional Chinese herbal medicine, can also activate PXR and induce CYP enzymes. Schisandrol B, Schisandrins A, and Schisandrins B were identified as the PXR-activating constituents isolated from WWZ [52]. All of these constituents showed similar potency and efficacy as RIF and Northern bolt analysis demonstrated that these constituents could induce both CYP3A and CYP2C9 mRNA expression. However, WWZ extracts have been used as a drug-sparing agent to decrease drug metabolism in the clinic. In one such example, co-administration of WWZ has been shown to increase the oral bioavailability of FK506 (Tacrolimus) [53]. WWZ or its constituent compounds have also been shown to increase the bioavailability of cyclosporine A and paclitaxel in animal models [54-56]. The metabolism of FK506 mainly occurred in the liver and small intestine via CYP3A [53]. FK506 is also a good substrate of the transporter p-gp [53]. It has been reported that WWZ extracts had an inhibitory effect on both CYP3A enzyme and p-gp. FK506 also undergoes a significantly first-pass effect and its gut availability is lower than the liver availability. WWZ can increase the gut availability of FK506 by about $111.4 \%$ and decrease the hepatic first-pass metabolism of FK506 [53]. The overall drug-sparing effect of WWZ suggests that the enzyme and transporter inhibitory effect of WWZ dominates the PXR-activating and enzymeinducing activity of the same herbal medicine.

Flavonoids represent another example of chemicals that have a dual effect in inhibiting DMEs and activating PXR. Flavonoids are commonly presented in plants, herbs and daily diets like blueberries, red wine, and grapefruit juice. All flavonoids except flavone inhibit CYP2C9 activity with the Ki value of less than $2.2 \mu \mathrm{M}$ [57]. Meanwhile, it has been reported that flavonoids, such as luteolin and apigenin, can activate
PXR-mediated CYP3A4 expression likely through the inactivation of several Cdk pathways [58]. In this case, the enzyme inhibitory effect is also dominating and, as a result, many drug labels warn patients to avoid drinking grapefruit juice when taking certain drugs. In the third example, the HIV protease inhibitor Ritonavir has been reported as both an inducer and inhibitor of CYP3A enzymes. In the clinic, Ritonavir has been widely used as a pharmacokinetic enhancer or boosting agent in HIV therapeutics [43]. Meclizine, an antihistamine to treat nausea, vomiting, and dizziness caused by motion sickness, is a human PXR agonist, but also an inhibitor of CYP3A4 [59].

\section{Summary}

The inhibition and induction of DMEs are issues of clinical importance, because they may cause drug-drug interactions or even undesirable drug toxicity. There are many clinical drugs that can function as DME inducers or inhibitors. Among the examples, diltiazem, a calcium-channel blocker, is a mechanism-based inactivator that forms metabolite intermediates and inhibits CYP enzyme. Rifampin, on the other hand, acts as a potent CYP inducer by activating PXR. When investigating drug-drug interactions, we should take both PXRmediated transcriptional induction and enzyme inhibition into account to prevent drug-drug interactions and side effects. Special caution should be given to drugs that have a narrow therapeutic index, such as the immunosuppressant Tacrolimus.

In vivo drug concentration is another important factor that determines whether PXR activation leads to increased drug metabolism. For example, simvastatin was ranked as having a high induction propensity based on the maximum induction and efficacy in vitro, and the mechanism of DME induction involves both CAR and PXR. However, simvastatin does not induce CYP3A4 at the maximum clinical dosage in vivo [44]. In another example of inconsistency between the in vitro and in vivo result, sulforaphane (SFN) is an isothiocyanate found

Fig. 1 Dual function of xenobiotics in PXR activation and CYP inhibition

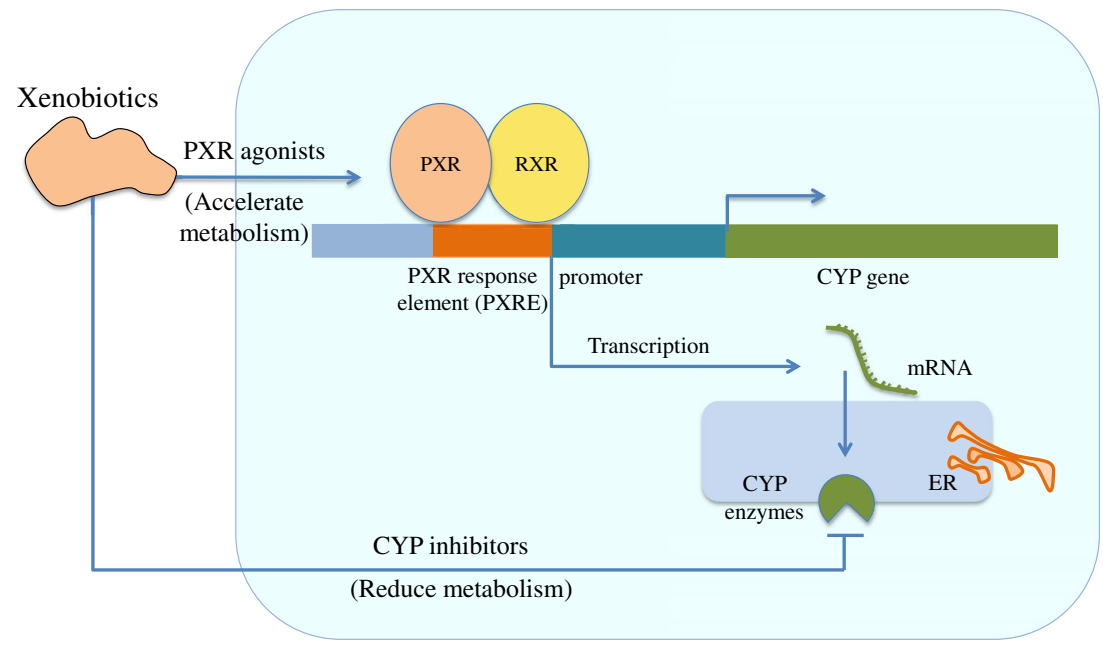


in broccoli that possesses an anticarcinogenic effect [60]. SFN was shown to be a PXR antagonist in human hepatocytes [61]. However, SFN did not exhibit a PXR antagonist effect in vivo, likely because the SFN-GSH conjugate is actively transported out of the liver, preventing the intracellular accumulation of SFN-GSH, or the SFN-GSH is unstable with a short half-life that cannot produce a persistent effect [60]. Therefore, we should consider whether in vitro data can be extrapolated to in vivo pharmacological effects. Even in vivo drug concentration may not precisely predict the intracellular concentration that the nuclear receptors are exposed to [45]. Last but not least, the PXR activation in rodent systems cannot be simply extrapolated to humans due to the species specificity of the PXR activation [14].

As summarized in Fig. 1, the net outcome depends on the balance between PXR induction and CYP inhibition, the pharmacokinetics of drugs and the in vivo drug concentrations, and whether the in vivo drug concentrations are high enough to produce an agonistic or antagonistic effect on PXR and thereby induce or inhibit DMEs.

\section{Compliance with Ethical Standards}

Conflict of Interest The authors declare no conflict of interest.

Human and Animal Rights and Informed Consent This article does not contain any studies with human or animal subjects performed by any of the authors.

\section{References}

1. Xie $\mathrm{W}$ et al. Reciprocal activation of xenobiotic response genes by nuclear receptors SXR/PXR and CAR. Genes Dev. 2000;14:3014-23.

2. Gao J, Xie W. Pregnane X receptor and constitutive androstane receptor at the crossroads of drug metabolism and energy metabolism. Drug Metab Dispos. 2010;38:2091-5.

3. Jancova P, Anzenbacher P, Anzenbacherova E. Phase II drug metabolizing enzymes. Biomed Pap. 2010;154:103-16.

4. Kliewer SA et al. An orphan nuclear receptor activated by pregnanes defines a novel steroid signaling pathway. Cell. 1998;92:73-82.

5. Blumberg B et al. SXR, a novel steroid and xenobiotic-sensing nuclear receptor. Genes Dev. 1998;12:3195-205.

6. Blumberg B et al. BXR, an embryonic orphan nuclear receptor activated by a novel class of endogenous benzoate metabolites. Genes Dev. 1998;12:1269-77.

7. Quattrochi LC, Mills AS, Barwick JL, Yockey CB, Guzelian PS. A novel cis-acting element in a liver cytochrome P450 3A gene confers synergistic induction by glucocorticoids plus antiglucocorticoids. J Biol Chem. 1995;270:28917-23.

8. Willson TM, Kliewer SA. PXR, CAR and drug metabolism. Nat Rev Drug Discov. 2002;1:259-66.

9. Goodwin B, Redinbo MR, Kliewer SA. Regulation of cyp3a gene transcription by the pregnane $\mathrm{x}$ receptor. Annu Rev Pharmacol Toxicol. 2002;42:1-23.

10. Chen $Y$ et al. Nuclear receptors in the multidrug resistance through the regulation of drug-metabolizing enzymes and drug transporters. Biochem Pharmacol. 2012;83:1112-26.
11. Watkins RE. The human nuclear xenobiotic receptor PXR: structural determinants of directed promiscuity. Science. 2001;292: 2329-33 (New York, NY).

12. Gillam EMJ. The PXR ligand-binding domain: how to be picky and promiscuous at the same time. Trends Pharmacol Sci. 2001;22:448.

13. Kim B et al. Global metabolomics and targeted steroid profiling reveal that rifampin, a strong human PXR activator, alters endogenous urinary steroid markers. J Proteome Res. 2013;12:1359-68.

14. Xie $\mathrm{W}$ et al. Orphan nuclear receptor-mediated xenobiotic regulation in drug metabolism. Drug Discov Today. 2004;9:442-9.

15. He J, Nishida S, Xu M, Makishima M, Xie W. PXR prevents cholesterol gallstone disease by regulating biosynthesis and transport of bile salts. Gastroenterology. 2011;140:2095-106.

16. Pondugula SR, Mani S. Pregnane xenobiotic receptor in cancer pathogenesis and therapeutic response. Cancer Lett. 2013;328:1-9.

17. Lee JY et al. Assessment of drug-drug interactions caused by metabolism-dependent cytochrome P450 inhibition. Chem Biol Interact. 2012;198:49-56.

18. Honkalammi J, Niemi M, Neuvonen PJ, Backman JT. Mechanismbased inactivation of CYP2C8 by gemfibrozil occurs rapidly in humans. Clin Pharmacol Ther. 2011;89:579-86.

19. Foti RS, Rock DA, Pearson JT, Wahlstrom JL, Wienkers LC. Mechanism-based inactivation of cytochrome P450 3A4 by mibefradil through heme destruction. Drug Metab Dispos. 2011;39:1188-95.

20. Lin JH, Lu AYH. Inhibition and induction of cytochrome P450 and the clinical implications. Clin Pharmacokinet. 2012;35:361-90.

21. Pelkonen $\mathrm{O}$ et al. Inhibition and induction of human cytochrome P450 enzymes: current status. Arch Toxicol. 2008;82:667-715.

22. Dudda A, Kuerzel G. In: Vogel HG, Maas J, Hock FJ, Mayer D, editors. Drug discovery and evaluation: safety and pharmacokinetic assays, vol. 44. Berlin: Springer; 2013. p. 989-1004.

23. Wang B. Drug-drug interactions in pharmaceutical development. Wiley-Interscience; 2008 .

24. Zhi-Yi Z, Wong YN. Enzyme kinetics for clinically relevant CYP inhibition. Curr Drug Metab. 2005;6:241-57.

25. Ring B, Wrighton S \& Mohutsky M. In: Nagar S, Argikar UA, Tweedie DJ editors. Enzyme kinetics in drug metabolism vol. 1113 methods in molecular biology. Humana Press; 2014 Ch. 4, p. $37-56$.

26. Kamel A, Harriman S. Inhibition of cytochrome P450 enzymes and biochemical aspects of mechanism-based inactivation (MBI). Drug Discov Today Technol. 2013;10:e177-89.

27. Wang X, Cheung CM, Lee WYW, Or PM Y, Yeung JHK. Major tanshinones of Danshen (Salvia miltiorrhiza) exhibit different modes of inhibition on human CYP1A2, CYP2C9, CYP2E1 and CYP3A4 activities in vitro. Phytomedicine. 2010;17:868.

28. Amit SK, Obach RS, Tristan SM. Mechanism-based inactivation of cytochrome P450 enzymes: chemical mechanisms, structureactivity relationships and relationship to clinical drug-drug interactions and idiosyncratic adverse drug reactions. Curr Drug Metab. 2007;8:407-47.

29. Lin J, Lu AH. Inhibition and induction of cytochrome P450 and the clinical implications. Clin Pharmacokinet. 1998;35:361-90.

30. VandenBrink BM, Isoherranen N. The role of metabolites in predicting drug-drug interactions: focus on irreversible P450 inhibition. urr Opin Drug Discov Dev. 2010;13:66-77.

31. Kunze KL, Mangold BL, Wheeler C, Beilan HS, de Montellano PR $\mathrm{O}$. The cytochrome P-450 active site. Regiospecificity of prosthetic heme alkylation by olefins and acetylenes. J Biol Chem. 1983;258: 4202-7.

32. Montellano PRO, Correia MA. Suicidal destruction of cytochrome P-450 during oxidative drug metabolism. Annu Rev Pharmacol Toxicol. 1983;23:481-503. 
33. Creek DJ et al. Relationship between antimalarial activity and heme alkylation for spiro- and dispiro-1,2,4-trioxolane antimalarials. Antimicrob Agents Chemother. 2008;52:1291-6.

34. Guengerich FP. Mechanism-based inactivation of human liver microsomal cytochrome P-450 IIIA4 by gestodene. Chem Res Toxicol. 1990;3:363-71.

35. Marks GS, McCluskey SA, Mackie JE, Riddick DS, James CA. Disruption of hepatic heme biosynthesis after interaction of xenobiotics with cytochrome P-450. FASEB J. 1988;2:2774-83.

36. Baer BR, DeLisle RK, Allen A. Benzylic oxidation of gemfibrozil1 -O- $\beta$-glucuronide by P450 2 C 8 leads to heme alkylation and irreversible inhibition. Chem Res Toxicol. 2009;22:1298-309.

37. Pratt-Hyatt M, Lin HL, Hollenberg PF. Mechanism-based inactivation of human CYP2E1 by diethyldithocarbamate. Drug Metab Dispos. 2010;38:2286-92.

38. He K, Woolf TF, Hollenberg PF. Mechanism-based inactivation of cytochrome P-450-3A4 by mifepristone (RU486). J Pharmacol Exp Ther. 1999;288:791-7.

39. Lin H-L, Kent UM, Hollenberg PF. Mechanism-based inactivation of cytochrome P450 3A4 by $17 \alpha$-ethynylestradiol: evidence for heme destruction and covalent binding to protein. J Pharmacol Exp Ther. 2002;301:160-7.

40. Yamaori S, Okushima Y, Yamamoto I, Watanabe K. Characterization of the structural determinants required for potent mechanism-based inhibition of human cytochrome P450 1A1 by cannabidiol. Chem Biol Interact. 2014;215:62-8.

41. Lu P et al. Mechanism-based inhibition of human liver microsomal cytochrome $\mathrm{p} 4501 \mathrm{a} 2$ by zileuton, a 5 -lipoxygenase inhibitor. Drug Metab Dispos. 2003;31:1352-60.

42. Cao J et al. Mechanism-based inactivation of cytochrome P450 2B6 by isoimperatorin. Chem Biol Interact. 2015;226:23-9.

43. Zheng $\mathrm{L}$ et al. Imperatorin is a mechanism-based inactivator of CYP2B6. Drug Metab Dispos. 2015;43:82-8.

44. Zhou $\mathrm{S}$ et al. Mechanism-based inhibition of cytochrome P450 3A4 by therapeutic drugs. Clin Pharmacokinet. 2005;44:279-304.

45. Barbara JE, Kazmi F, Parkinson A, Buckley DB. Metabolismdependent inhibition of CYP3A4 by lapatinib: evidence for formation of a metabolic intermediate complex with a nitroso/oxime metabolite formed via a nitrone intermediate. Drug Metab Dispos. 2013;41:1012-22

46. Sunaga $\mathrm{K}$ et al. Mechanism-based inhibition of recombinant human cytochrome P450 3A4 by tomato juice extract. Biol Pharm Bull. 2012;35:329-34.

47. Greenblatt DJ et al. Mechanism-based inhibition of human cytochrome P450-3A activity by grapefruit hybrids having low furanocoumarin content. Xenobiotica. 2012;42:1163-9.
48. Quattrochi LC, Guzelian PS. Cyp3A regulation: from pharmacology to nuclear receptors. Drug Metab Dispos. 2001;29:615-22.

49. Savas U, Griffin KJ, Johnson EF. Molecular mechanisms of cytochrome P-450 induction by xenobiotics: an expanded role for nuclear hormone receptors. Mol Pharmacol. 1999;56:851-7.

50. Guengerich FP. Cytochrome P-450 3A4: regulation and role in drug metabolism. Annu Rev Pharmacol Toxicol. 1999;39:1-17.

51. Li F et al. Human PXR modulates hepatotoxicity associated with rifampicin and isoniazid co-therapy. Nat Med. 2013;19:418-20.

52. $\mathrm{Mu} \mathrm{Y}$ et al. Traditional Chinese medicines Wu Wei Zi (Schisandra chinensis Baill) and Gan Cao (Glycyrrhiza uralensis Fisch) activate pregnane $\mathrm{X}$ receptor and increase warfarin clearance in rats. $\mathrm{J}$ Pharmacol Exp Ther. 2006;316:1369-77.

53. Qin XL et al. Mechanistic understanding of the different effects of Wuzhi Tablet (Schisandra sphenanthera extract) on the absorption and first-pass intestinal and hepatic metabolism of Tacrolimus (FK506). Int J Pharm. 2010;389:114-21.

54. Jin J et al. Effect of Wuzhi tablet (Schisandra sphenanthera extract) on the pharmacokinetics of paclitaxel in rats. Phytother Res. 2011;25:1250-3.

55. Jin $\mathrm{J}$ et al. Enhancement of oral bioavailability of paclitaxel after oral administration of Schisandrol B in rats. Biopharm Drug Dispos. 2010;31:264-8.

56. Qin XL et al. In vivo to in vitro effects of six bioactive lignans of Wuzhi tablet (Schisandra sphenanthera extract) on the CYP3A/Pglycoprotein-mediated absorption and metabolism of Tacrolimus. Drug Metab Dispos. 2014;42:193-9.

57. Si D et al. Mechanism of CYP2C9 inhibition by flavones and flavonols. Drug Metab Dispos. 2009;37:629-34.

58. Dong $\mathrm{H}$, Lin $\mathrm{W}, \mathrm{Wu} \mathrm{J}$, Chen $\mathrm{T}$. Flavonoids activate pregnane $\mathrm{x}$ receptor-mediated CYP3A4 gene expression by inhibiting cyclindependent kinases in HepG2 liver carcinoma cells. BMC Biochem. 2010;11:23

59. Foo WYB, Tay HY, Chan ECY, Lau AJ. Meclizine, a pregnane X receptor agonist, is a direct inhibitor and mechanism-based inactivator of human cytochrome P450 3A. Biochem Pharmacol. 2015;97:320-30.

60. Poulton EJ et al. Sulforaphane is not an effective antagonist of the human pregnane X-receptor in vivo. Toxicol Appl Pharmacol. 2013;266:122-31.

61. Zhou $\mathrm{C}$ et al. The dietary isothiocyanate sulforaphane is an antagonist of the human steroid and xenobiotic nuclear receptor. Mol Pharmacol. 2007;71:220-9. 